\title{
Impact of Defect Creation And Motion on the Thermodynamics and Large-Scale Reorganization of Self-Assembled Clathrin Lattices
}

\author{
Shafigh Mehraeen ${ }^{a}$, Nicholas Cordella ${ }^{b c}$, Jee Soo Yoo ${ }^{c d}$ and Andrew J. Spakowitz ${ }^{* c e}$ \\ ${ }^{a}$ Department of Mechanical Engineering, Stanford University, Stanford, CA 94305, USA \\ ${ }^{b}$ Department of Chemical Engineering, Stanford University, Stanford, CA 94305, USA
}

${ }^{c}$ Stanford Institute for Materials and Energy Sciences (SIMES), SLAC National Accelerator Laboratory, 2575 Sand

Hill Road, Menlo Park, CA 94025, USA

${ }^{d}$ Department of Materials Science and Engineering, Stanford University, Stanford, CA 94305, USA

${ }^{e}$ Department of Chemical Engineering, Stanford University, Stanford, CA 94305, USA. E-mail:

ajspakow@stanford.edu; Fax: +(650) 724-0451; Tel: +(650) 736-8733

Received 13th January 2011 , Accepted 4th April 2011

First published on the web 23rd May 2011

We develop a theoretical model for the thermodynamics and kinetics of clathrin self-assembly. Our model addresses the behavior in two dimensions and can be easily extended to three dimensions, facilitating the study of membrane, surface, and bulk assembly. The clathrin triskelia are modeled as flexible pinwheels that form leg-leg associations and resist bending and stretching deformations. Thus, the pinwheels are capable of forming a range of ring structures, including 5-, 6-, and 7-member rings that are observed experimentally. Our theoretical model employs Brownian dynamics to track the motion of clathrin pinwheels at sufficiently long time scales to achieve complete assembly. Invoking theories of dislocation-mediated melting in two dimensions, we discuss the phase behavior for clathrin selfassembly as predicted by our theoretical model. We demonstrate that the generation of 5-7 defects in an otherwise perfect honeycomb lattice resembles creation of two dislocations with equal and opposite Burgers vectors. We use orientational- and translational-order correlation functions to predict the crystalline-hexatic and hexatic-liquid phase transitions in clathrin lattices. These results illustrate the pivotal role that molecular elasticity plays in the physical behavior of self-assembling and self-healing materials.

\section{Introduction}

Many biological systems are capable of spontaneously assembling a diverse set of molecular architectures from a single subunit. Clathrin-mediated endocytosis involves the formation of a pit that is surrounded by a honeycomb coating whose pinwheel-shaped (or triskelion) subunit is a clathrin-protein complex. This characteristic ability of clathrin has

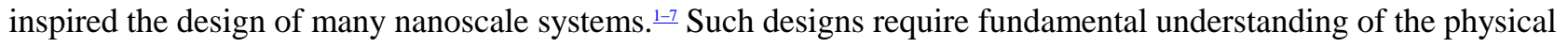


principles involved in biological self-assembly in order to fully exploit the inherent adaptability of such soft-matter systems for responsive and self-healing materials applications.

The triskelion structure of the clathrin subunit facilitates the formation of nanoscale cages and pits $\stackrel{8.9}{-}$ upon recruitment of clathrin to the cell wall by adaptor proteins..$^{10}$ These cage-like lattices contain a clathrin subunit at each vertex, and the three triskelion arms associate with neighboring triskelia via favorable heavy- and light-chain interactions. $\frac{8.9}{}$ Thus, the resulting lattice has three bonds at each vertex when fully satisfied, resulting in a honeycomb structure with prevalent five-membered and seven-membered ring defects. As in any closed polyhedral lattice, such ring defects permit closure of the lattice into a three-dimensional shell. Clathrin achieves this via the formation of a pit that comprises the seed of an endosomal vesicle. ${ }^{11.12}$ Two and three dimensional clathrin assemblies are also observed in vitro, ${ }^{13-16}$ indicating that these assemblies can be highly variable.

The elastic flexibility of the clathrin triskelion plays a critical role in facilitating the formation of a wide range of architectures both in vivo and in vitro. ${ }^{17-20}$ Structural analysis of clathrin triskelia reveals the orientation of the flexible legs $\mathrm{s}^{13}$ by positioning the triskelia in experimentally observed lattices. ${ }^{21.22}$ The size distribution of cages, discerned from electron micrographs, $\frac{18.19}{19}$ is used to estimate rigidity of clathrin legs. Statistical analysis of light scattering data reveals independent structural fluctuations of triskelion legs. $\underline{20}$

Clathrin provides us with a useful system to understand the pivotal role of elasticity, molecular interactions, and local structural rearrangement in the behavior of self-assembled materials. Structural rearrangement in polycrystalline materials leads to the generation and migration of grain boundaries ${ }^{23}$ and can influence phase transitions of crystals by inducing geometrical and topological defects. ${ }^{24}$ In ordered structures on curved surfaces, ${ }^{25.26}$ local structural changes lead to defect formation to alleviate internal elastic stresses. Understanding the physical mechanisms underlying selfassembled systems provides insight on how to externally control the assembly of nanoscale building blocks into materials with favorable optical, electronic, and mechanical properties. ${ }^{27}$ Such physical insight can also inspire the fabrication of switchable nanomaterials,,$\frac{28}{}$ semiconductor sheets of nano-particles,, 29 and self-healing materials. ${ }^{30}$

Our goal in this manuscript is to develop a two-dimensional model of clathrin that captures the physical effects dictating large-scale reorganization due to local structural rearrangements. This coarse-grained model permits us to perform numerical simulations of clathrin assembly dynamics at sufficient time and length scales to address the thermodynamics and kinetics of biologically relevant lattice structures. We then proceed to determine bulk elastic constants for an effective continuum solid and map our model onto classical theories of dislocation-mediated melting in two dimensions. $\frac{31-34}{}$ Our model offers new insight into the role of local molecular elasticity in the large-scale response of self-assembled architectures. This framework can be easily extended to address three-dimensional and membranebound clathrin self-assembly.

Section 2 describes our theoretical model. We discuss our results in Sec. 3, focusing on the role of dislocations in the structural dynamics of a clathrin lattice. We conclude in Sec. 4 by summarizing our findings and elaborate on future research goals. 


\section{Theoretical Model}

In this section, we develop a two-dimensional mesoscale model of clathrin. The individual clathrin protein complex takes the form of a pinwheel composed of three legs connected to a vertex (see Fig. 1). The vertex position $\vec{r}_{i}$ gives the location of the $i$ th pinwheel, and the orientation of the legs are dictated by the vectors adjoining bonded pinwheel pairs to each other. Therefore, the leg orientations are defined by the pinwheel coordinates and the bond connections.

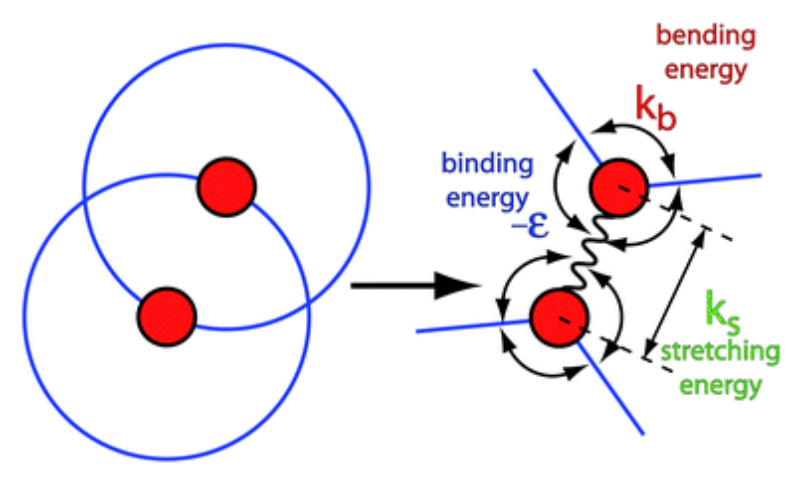

Fig. 1 Two-dimensional mesoscale model of clathrin triskelion, indicating the energy change for transitioning from two unbound pinwheels (left, blue rings indicate clathrin leg length $r_{0}$ ) to two bound pinwheels (right). This schematic introduces the bending modulus $k_{b}$, stretching modulus $k_{s}$, and the binding affinity $\varepsilon$

Elastic energy contributions from leg deformations include stretching and bending energies, modeled as Hookean

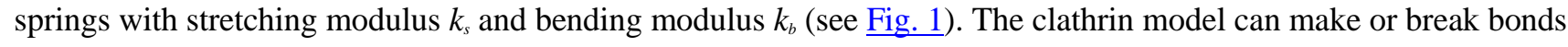
with neighbors via binding or unbinding events. Each bond contributes an energy $-\varepsilon$ to the total energy. In addition to bending, stretching, and binding energies, we consider a repulsive potential energy to avoid the overlapping of pinwheels. A detailed mathematical development of our clathrin model is provided in Appendix A, where we provide explicit definitions of the energetic contributions and the binding connectivity.

To simulate the dynamic behavior of our clathrin model, we combine Brownian dynamics with dynamic Monte Carlo simulations to track the motion of pinwheels and the evolving binding connectivity. This novel simulation approach tracks the dynamic evolution of the bead positions and bonds between pinwheels at sufficient time and length scales to permit large-scale assembly of lattices, as shown in Sec. 3. The development of the equations governing the pinwheel motion and the binding kinetics is found in Appendix B.

Our discrete model can be mapped onto an effective continuum elasticity model. This picture is adequate to address large-scale mechanical deformation energy and thermodynamic phenomena, which is exploited in Sec. 3. The elasticity of a continuum solid is defined by the Lamé coefficients of the material. In Appendix C, we derive the effective Lamé coefficients ${ }^{40.41}$ for a fully satisfied honeycomb lattice with no vacancies. 
From our derivations in Appendix C, we arrive at the Lamé coefficients

$$
\begin{aligned}
& \lambda=\frac{k_{s}}{4 \sqrt{3}}-\frac{\sqrt{3} k_{b}}{2 r_{0}^{2}}, \\
& \mu=\frac{k_{s}}{4 \sqrt{3}}+\frac{\sqrt{3} k_{b}}{2 r_{0}^{2}} .
\end{aligned}
$$

These elastic constants govern elastic deformation of the lattice over lengths that are large in comparison to the discretization length $r_{0}$ (i.e. the clathrin equilibrium leg length). The Lamé coefficients for our two-dimensional system are recast as the Young's modulus $E$ and Poisson's ratio $v$, such that ${ }^{42}$

$$
\begin{gathered}
E=\frac{4 \mu(\lambda+\mu)}{\lambda+2 \mu}=\frac{2 k_{s}}{3 \sqrt{3}} \frac{6+\alpha}{2+\alpha}, \\
v=\frac{\lambda}{\lambda+2 \mu}=\frac{1}{3}-\frac{8}{6+3 \alpha},
\end{gathered}
$$

where $\alpha=r_{0}^{2} k_{s} / k_{b}$. Our theoretical prediction of the Lamé coefficients are purely based on elastic mechanics of a perfect honeycomb lattice. These act as a starting point for addressing the physical behavior of a fluctuating lattice containing defects, which will be addressed in more detail in Sec. 3 when we consider the phase behavior of a clathrin lattice.

\section{Discussion}

Our model permits an examination of the effect of concentration, elasticity, and binding affinity on clathrin selfassembly on a two-dimensional surface or rigid membrane. We present simulations and theories for the dynamics of assembly and the structural fluctuations of an assembled lattice, addressing physical issues that are relevant to the formation and reorganization of a clathrin film. Our goal is to reveal the fundamental mechanisms that are essential in determining the large-scale behavior of an assembled lattice based on properties of the individual subunits. Such collective effects are at the heart of the biological function of clathrin during endocytosis.

We present in Fig. 2 a Brownian dynamic simulation of the progression of structural states that arise during the assembly of a clathrin lattice. We obtain the time history of 500 pinwheels in a box with side length equal to $27 r_{0}$ and periodic boundary conditions over 200 time steps, where one time step is equivalent to the time scale for a pinwheel to diffuse a distance equal to the bond size $r_{0}$. Fig. 2 shows 6 snapshots taken at times $t=1,2,10,20,100,200$. The simulation in Fig. 2 has bending and stretching moduli, $\beta k_{b}=5$ and $\beta k_{s} r_{0}{ }^{2}=50$ [where $\beta=1 /\left(k_{B} T\right)$ ], and the strength of the repulsive potential $\beta k_{l} / r_{0}{ }^{4}=1$. These snapshots represent a slow quench, where the binding affinity increases linearly from $\varepsilon=2.75 k_{B} T$ to $3.75 k_{B} T$ in $t=200$ time steps. The initial condition for this simulation has all of the pinwheel legs free and pinwheel positions that are random and uniformly distributed throughout the box, i.e. starting from an ideal-gas state. 


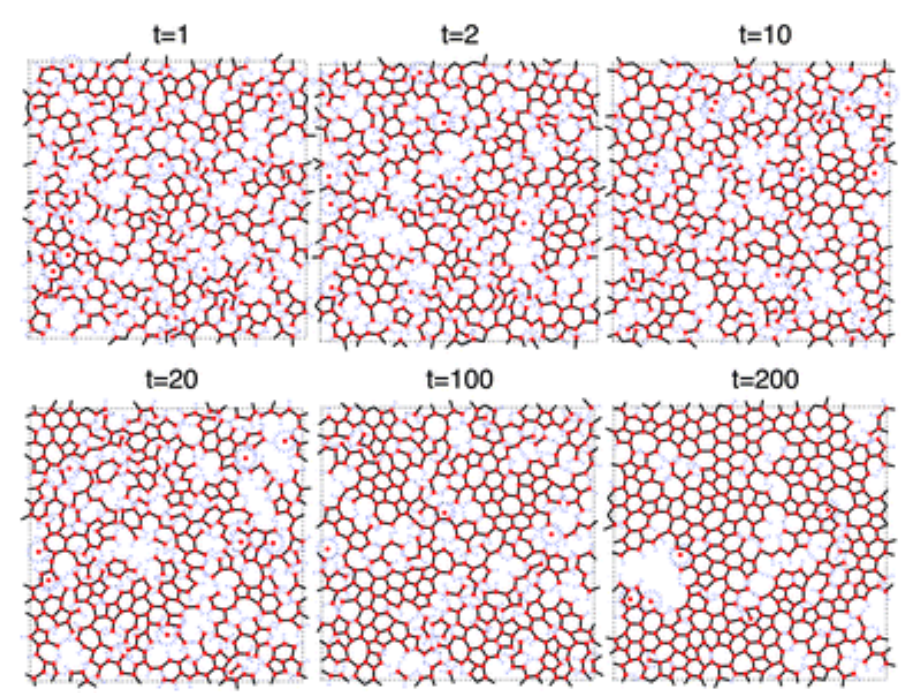

Fig. 2 Brownian dynamic simulation of the assembly dynamics of 500 pinwheels, with binding affinity $\varepsilon$ ramping up linearly from $2.75 k_{B} T$ to $3.75 k_{B} T$ in 200 time steps. Red dots identify the centers of pinwheels, and solid black and dashed blue segments are the bound and free legs, $r$

Close inspection of the assembly dynamics in Fig. 2 demonstrates an initial prevalence of small clusters and chains. Local structure favors 6-member rings; though, leg flexibility permits a range of ring sizes throughout the assembly. At this high density, the initial clusters quickly coalesce into a percolated structure within 2 time steps. The initial macrostructure at the point of percolation $(t=2)$ arises from clusters and chains of a range of size and shape, resulting in a structure that contains a broad distribution of ring and vacancy defects that induce considerable elastic frustration within the assembled structure. Subsequent reorganization to the final assembled state involves a series of lattice manipulations that alleviate the elastic frustration. The progression of lattice reorganization in Fig. 2 results in internal void regions toward which defects migrate, leaving a final structure $(t=200$ in Fig. 2$)$ with local orientational order and pinwheel legs that are mostly satisfied.

Brownian dynamic simulations illustrate the effect of leg stiffness, binding affinity, and quench rate on the dynamics of self assembly and the morphology of the assembled lattice. Defects in this structure are defined by nonhexagonal rings, especially pentagons and heptagons, within the ground-state honeycomb lattice. Although the flexibility of the clathrin triskelia permits a lattice to accommodate ring defects, the kinetic processes that underlie rearrangement of the lattice ultimately dictate whether the lattice is capable of achieving a dynamic response to local stimuli. For example, the formation of a lattice with a single isolated five-membered ring could occur via a large-scale excision of a $60^{\circ}$ lattice wedge..$^{43}$ This mechanism becomes energetically prohibitive for large lattice patches.

Alternatively, an isolated five-membered ring can be formed through a series of local lattice reorganizations that result in the large-scale separation of five- and seven-membered ring defects. ${ }^{44}$ Along these lines, the energetics of defect creation and separation dictate the capacity for the lattice to undergo rearrangement. We now discuss the creation, annihilation, migration, and interaction of defects in an otherwise perfect honeycomb structure, addressing the impact of defects on the phase behavior and macroscale properties of an assembled lattice. 
Creation and annihilation of defects within an assembled lattice can occur through local bond reorganization events. Such topological transitions arise in isomerization processes in fullerenes. ${ }^{45}$ The top image of Fig. 3 demonstrates that a bond rotation event within a perfect honeycomb lattice generates two sets of 5- and 7-member rings, i.e. two 5-7 defects. Adjacent 5-member (red pentagon) and 7-member (blue heptagon) rings are also frequently seen in our Brownian dynamic simulations (bottom left) as well as in cryo-EM images ${ }^{14}$ (bottom right) of clathrin assemblages. Notably, the cryo-EM image in Fig. 3 contains prevalent instances where 5-member and 7-member rings are localized together, as would arise from defect creation processes and from elastic considerations (discussed further below). Assembled structures from our simulations contain square (yellow), octagon (green), and more energetically unfavorable defect structures as well as frequent 5-7 pairs. The patterns of emerging defects due to local reorganization depend on the configuration of the patch in which the reorganization occurs. For instance, if we find a patch where two hexagons and two pentagons initially meet in a crisscross pattern, rotation of the central bond only swaps pentagons and hexagons in a Stone-Wales rearrangement. ${ }^{45}$ Such a rearrangement does not create new pentagons.

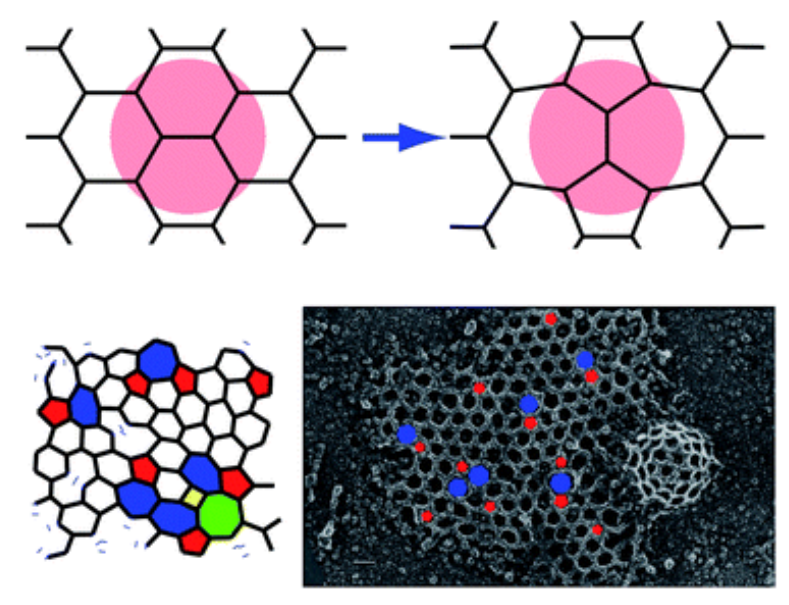

Fig. 3 The top image shows the lattice-reorganization mechanism to generate two 5-7 defects in a honeycomb lattice while maintaining all bonds. The bottom images show the existence of 5-7 defects in both a lattice obtained from Brownian dynamic simulation (bottom, left) and a clathrin lattice obtained experimentally (bottom, right)..$^{14}$

The defect-creation process (top image of Fig. 3) can occur via several mechanisms, one of which is shown in the top image of Fig. 4. The formation of a pair of 5-7 defects occurs through a sequence of steps (labeled with reaction coordinate $0, \ldots, 7, F)$, each of which involves either unbinding of a bound leg (solid line) or binding of a free leg (dashed line). Each image in Fig. 4 is rendered from a numerical optimization of our model with the appropriate leg connectivities for each step. The corresponding total energy change for this sequence of steps as a function of reaction coordinate is shown in the bottom of Fig. 4 for binding affinity $\varepsilon=2.5$ (red), 3.5 (green), 4.5 (blue), and 5.5 $k_{B} T$ (black), stretching modulus $\beta k_{s} r_{0}{ }^{2}=50$, and bending modulus $\beta k_{b}=5$. Through this particular pathway, the creation of a pair of 5-7 defects for binding affinity $\varepsilon=2.5 k_{B} T$ requires overcoming an energy barrier of $\sim 10 k_{B} T$. This barrier increases to $\sim 16.5 k_{B} T$ for binding affinity $\varepsilon=5.5 k_{B} T$, resulting in a considerable energetic barrier that renders such events kinetically limited. Defining the Burgers vector of a defect as the vector drawn from the heptagon center to the 
pentagon center and rotated by $90^{\circ}$ counter-clockwise, ${ }^{46}$ the Burgers vectors of the two 5-7 defects in the final state $F$ sum to zero; this sum is a conserved quantity for any internal lattice reorganization, as in Fig. 4.
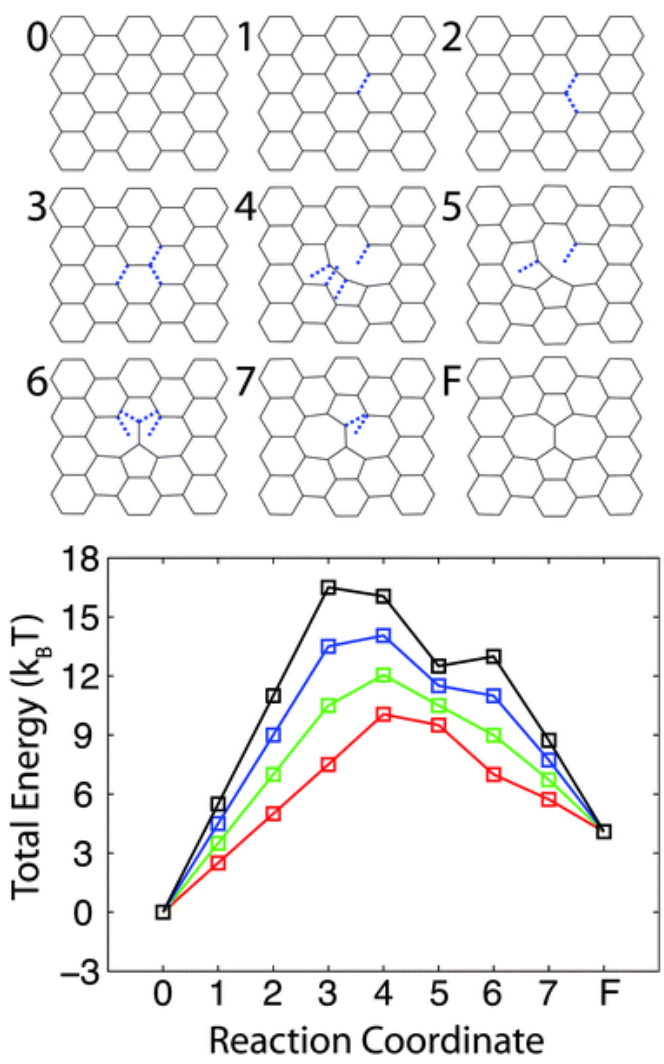

Fig. 4 The top series of images shows a step-by-step mechanism for the creation of two 5-7 defects in a honeycomb lattice. The bottom figure gives the total energy change versus reaction coordinate. The energy barrier to generate two 5-7 defects increases with binding affinity $\varepsilon=2.5$ (red), 3.5 (green), 4.5 (blue), and $5.5 k_{B} T$ (black).

Results presented in Fig. 4 suggest that binding affinity plays a pivotal role in defect-generation kinetics by dictating the barrier for lattice reorganization. However, the energy of the initial and final states in Fig. 4 are insensitive to the binding affinity, since all legs are bonded in these states. The transition to the final state $F$ results in a core energy $E_{c}$ of the defect pair, representing the energetic cost for defect creation. This cost is offset by the entropic benefit associated with translation of the defects throughout the lattice. Defect translation is a critical determinant of the thermodynamic behavior and the mechanical properties of the lattice.

Upon creation of a pair of 5-7 defects via a mechanism typified in Fig. 4, the two defects can be separated from each other by a series of lattice reorganizations akin to those shown in Fig. 3 and $\underline{4}$. For example, the horizontal bond at the top of the right-side heptagon can be rotated by $90^{\circ}$ within the lattice, resulting in the upper-right 5-7 defect shifting within the lattice up and to the right by one unit. This translation step is shown in Panel A of Fig. 5, along with 
subsequent reorganization processes that further separate the defect pair. No new defects are created throughout the translation process in Fig. 5A, and the sum of the two Burgers vectors remains zero.
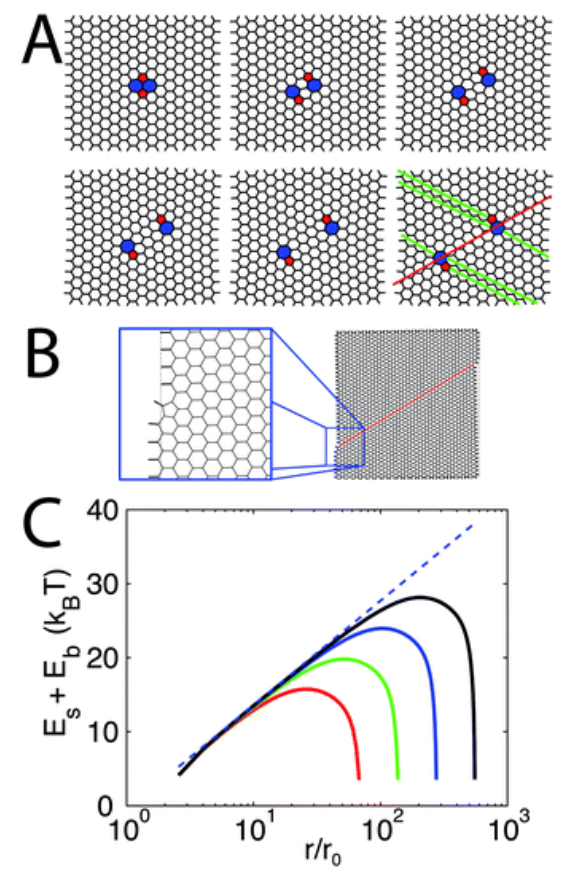

Fig. 5 Panel A shows minimum-energy configurations of a honeycomb lattice $\left(B k_{s} r_{0}{ }^{2}=50\right.$ and $\left.B k_{b}=5\right)$ as two 5-7 defects are separated. Panel $B$ demonstrates that defect separation leads to large-scale lattice reorganization along a slip line (red) within the lattice. Panel $C$ gives the elastic energy $E_{s}+E_{b}$ as a function of separation distance, obtained from numerical optimization with 3000 (red), 12000 (green), 45000 (blue), and 180000 (black) pinwheels and from analytical theory based on continuum mechanics (dashed line).

The bottom-right image in Fig. 5A labels the hexagonal columns entering and exiting each defect, demonstrating an analogy between the defect structure within the honeycomb lattice and two parallel edge dislocations with equal and opposite Burgers vectors. A column of hexagons entering the defect is split in two upon exiting from the defect, akin to an edge dislocation where a half plane of atoms is inserted in a perfect crystal. The slip line (i.e. a slip plane in two dimensions) associated with such an edge dislocation is illustrated by separating the 5-7 defects all the way to the edges of the honeycomb lattice as shown in Fig. 5B. The slip line is shown as a red connecting line between the two defects across the lattice. Fig. 5B demonstrates that defect separation results in large-scale lattice reorganization that incurs fluidity to the macrostructure, suggesting that defect creation and translation are essential in permitting the lattice to be structurally responsive.

Panel $\mathrm{C}$ of Fig. 5 gives the total elastic energy $E_{s}+E_{b}$ versus separation distance between two defects for $\beta k_{s} r_{0}^{2}=50$ and $\beta k_{b}=5$. The solid curves represent the total energy obtained from numerical optimization of our model for 3000 (red), 12000 (green), 45000 (blue), and 180000 (black) pinwheels. Our numerical optimization finds the minimumenergy configuration for a square patch of pinwheels with free edges (i.e. no periodic boundary conditions), thus eliminating the interactions between defects through lattice periodicity. The dashed blue curve is a theoretical 
prediction from continuum elasticity theory, ${ }^{47}$ where the elastic energy of an isotropic continuum with two parallel edge dislocations is given by

$$
\begin{aligned}
E_{\text {dssloc }} & =\frac{b^{2}}{\pi} \frac{\mu(\lambda+\mu)}{(\lambda+2 \mu)}(\log r+c) \\
& =\frac{\sqrt{3}}{18 \pi} b^{2} k_{s} \frac{6+\alpha}{2+\alpha}(\log r+c) \\
& =\frac{k_{B} T}{4 \pi} K(\log r+c),
\end{aligned}
$$

where $\alpha=r_{0}^{2} k_{s} / k_{b}$. Here, $\mu$ and $\lambda$ are the Lamé coefficients, $r$ is the separation distance between defects, $b=\sqrt{3} r_{0}$ is the magnitude of Burgers vector, and $c$ is a constant related to the orientation of the line connecting the two edge dislocations. ${ }^{47}$ The second form of $E_{\text {disloc }}$ in eqn (5) utilizes our analytical results from Sec. 2 for the Lamé coefficients, and the blue dashed curve in Fig. $5 \mathrm{C}$ gives this result with a single additive constant to match the simulation results. The third form of $E_{\text {disoc }}$ in eqn (5) defines the dislocation coupling constant $K$. Fig. 5C confirms that the elastic energy of two 5-7 defects in an otherwise perfect honeycomb lattice follows the logarithmic behavior described in eqn (5) at large separation.

Our current mechanical analyses lay the foundation for a discussion of the thermodynamic behavior of our clathrin model. We begin by presenting Monte Carlo simulations of our model with various values of the elastic moduli $k_{s}$ and $k_{b}$. These simulations involve 1972 pinwheels in a box of size $51 r_{0}$ by $50.2 r_{0}$ with periodic boundary conditions. These box dimensions align with a fully-satisfied, undeformed honeycomb lattice with 1972 pinwheels. We seed each simulation with a perfect honeycomb lattice configuration in which all pinwheel legs are initially satisfied and perform a total of $6 \times 10^{7}$ Monte Carlo steps. Each Monte Carlo step consists of two stages. In the first stage, a randomly selected pinwheel is randomly translated without making or breaking bonds. We accept or reject the trial configuration based on a Metropolis selection criterion,,$^{37}$ i.e. a probability based on Boltzmann distributed density function incorporating the elastic and repulsive energy change. In the second stage, we randomly select a leg from the pinwheel chosen in the first stage. If the leg is bound, we attempt to unbind the leg. If the leg is unbound, we attempt to bind the leg to a nearby available free leg. We accept or reject the second trial configuration using Boltzmann distributed probability density function of the accompanying elastic energy and leg-leg interaction energy change.

Final snapshots from Monte Carlo simulations of 1972 pinwheels are shown in Fig. 6 for stretching modulus $\beta k_{s} r_{0}{ }^{2}=$ 85 (A), 75 (B), 70 (C), 65 (D), 60 (E), and 55 (F), where the bending modulus is $\beta k_{b}=\beta k_{s} r_{0}{ }^{2} / 10$. In these snapshots, 5 member and 7-member rings are colored red and blue, respectively. Our simulations shown in Fig. 6 are sufficiently large such that interactions between separate defects are far more prevalent than interactions between defects and their own images (even for case A), thus rendering the effects of periodic boundary conditions negligible. These images demonstrate that pinwheel rigidity plays a critical role in the propensity for the lattice to form defects and in the tendency for these defects to remain bound to each other. For large rigidity $\left(\beta k_{s} r_{0}^{2}=85\right.$, Fig. 6A), the lattice contains a low concentration of 5-7 defects that tend to be adjacent to each other, as in the first image of Fig. $5 \mathrm{~A}$. As the 
stretching and bending moduli are reduced, the defect concentration increases, and the defects tend to separate due to structural fluctuations of the lattice. Moderate values of $\beta k_{s} r_{0}{ }^{2}$ (i.e.Fig. 6C to Fig. 6D) tend to have substantial separation of the defects; however, the 5-7 defects remain intact, rather than separating into isolated 5-member and 7-member rings. Further reduction of $\beta k_{s} r_{0}{ }^{2}$ (i.e. Fig. 6E to Fig. 6F) leads to large concentration of defects that easily separate from each other and that split into distinct 5-member and 7-member rings within the lattice. These qualitative observations underscore a complex relationship between the microscopic pinwheel elasticity and the macroscopic thermodynamic behavior that can be addressed in terms of the phase behavior of two-dimensional crystals.

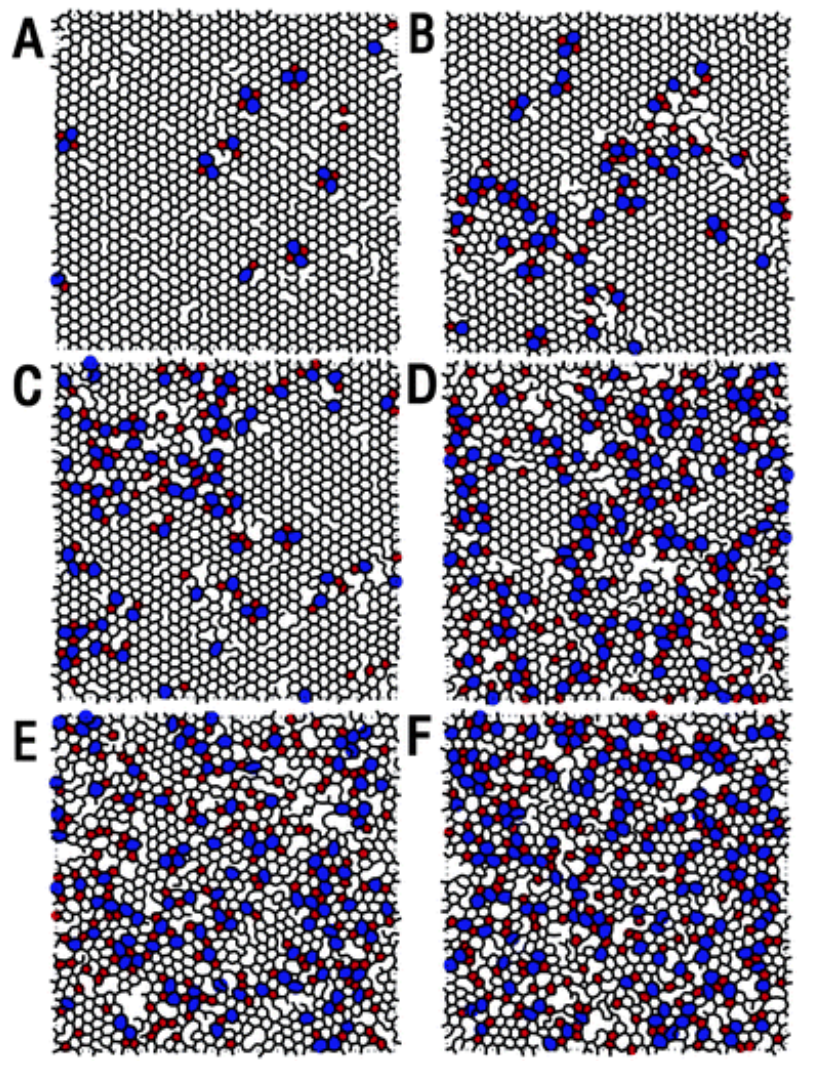

Fig. 6 Snapshots from Monte Carlo simulations of a clathrin lattice, demonstrating the effect of elasticity $\left[6 k_{s} r_{0}^{2}=85(\mathrm{~A}), 75(\mathrm{~B}), 70(\mathrm{C}), 65(\mathrm{D}), 60(\mathrm{E})\right.$, and $55(\mathrm{~F})$ and $\left.8 k_{b}=6 k_{s} r_{0}^{2} / 10\right]$ on large-scale structural fluctuations. To locate defects, pentagons and heptagons are shown in red and blue, respectively.

Classical theories of dislocation-mediated melting in two dimensions (KTNHY theory) ${ }^{31-34}$ propose that dislocations in the ordered crystalline phase tend to unbind upon raising the temperature, leading to isolated and mobile dislocations in a hexatic phase. Fig. 5B demonstrates that defect separation leads to large-scale lattice reorganization; thus, embedding many mobile defects in the lattice leads to a structure with fluid-like mechanical properties. In our clathrin model, a 5-7 defect is composed of 5-member and 7-member disclinations. Further increase in temperature enhances the entropic benefit of separation of dislocations into their disclinations, leading to a phase transition to a disordered liquid state. 
The interactions between dislocations in a fluctuating lattice are modulated by the structural fluctuations within the environment surrounding the dislocations. Kosterlitz and Thouless expressed this in terms of a dielectric medium surrounding the dislocations, $\frac{31}{}$ and Nelson, Halperin, and Young utilized Renormalization Group theory to determine the macroscale properties that dictate the effective interactions between dislocations. ${ }^{32-34}$ In order to precisely find the conditions for the crystalline-hexatic phase transition, we look at renormalized properties using the KTNHY theory recursion relations $\frac{32-34}{24}$

$$
\begin{aligned}
\frac{\partial K_{R}^{-1}}{\partial l} & =3 \pi y_{R}^{2} \exp \left(\frac{K_{R}}{8 \pi}\right)\left[\frac{1}{2} I_{0}\left(\frac{K_{R}}{8 \pi}\right)-\frac{1}{4} I_{1}\left(\frac{K_{R}}{8 \pi}\right)\right], \\
\frac{\partial y_{R}}{\partial l} & =\left(2-\frac{K_{R}}{8 \pi}\right) y_{R}+2 \pi y_{R}^{2} \exp \left(\frac{K_{R}}{16 \pi}\right) I_{0}\left(\frac{K_{R}}{8 \pi}\right), \\
\frac{\partial \mu_{R}^{-1}}{\partial l} & =3 \pi y_{R}^{2} \exp \left(-\frac{K_{R}}{8 \pi}\right) I_{0}\left(\frac{K_{R}}{8 \pi}\right) .
\end{aligned}
$$

Eqn (6) governs the renormalized dislocation coupling constant $K_{R}$, the renormalized fugacity of a dislocation pair $y_{R}\left(i . e\right.$. the effective dislocation probability), and the renormalized shear modulus of the clathrin lattice $\mu_{R}$ for varying reduced linear dimension $l=\log (r / b)$ (with ${ }^{b=\sqrt{3}} r_{0}$ ). In eqn (6), $I_{0}$ and $I_{1}$ are modified Bessel functions of the first kind. The macroscale properties of the lattice are found by integrating eqn (6) from $l=0$ to $l \rightarrow \infty$, subject to the initial conditions $K_{R}(l=0)=K, y_{R}(l=0)=\exp \left(-\beta E_{c}\right)$, and $\mu_{R}(l=0)=\mu$, where $E_{c}$ is the core energy of the defect pair, $\mu$ is the bare shear modulus (derived in Sec. 2), and the bare coupling constant $K$ is given in eqn (5).

Fig. 7 shows the renormalized dislocation coupling constant $K_{R}$ versus $\beta k_{s} r_{0}^{2}$ (with $k_{b}=k_{s} r_{0}^{2} / 10$ ) by solving the renormalization group differential equations in eqn (6) as $l$ tends to infinity. According to Fig. $7, K_{R}$ decreases and approaches a universal constant $16 \pi$ as stretching modulus $\beta k_{s} r_{0}{ }^{2}$ approaches 69 from above. At this point, the coupling constant $K_{R}$ jumps discontinuously to zero. According to the Kosterlitz-Thouless criterion for melting,, the renormalized Lamé coefficient $\mu_{R}$ is also discontinuous across the melting point, as shown in the inset of Fig. 7. The discontinuous drop of dislocation coupling constant $K_{R}$ and Lamé coefficient $\mu_{R}$ at $\beta k_{s} r_{0}^{2}=69$ indicates a crystallinehexatic phase transition, where the system goes from a solid phase to a liquid-like hexatic phase that does not resist shear deformation. 


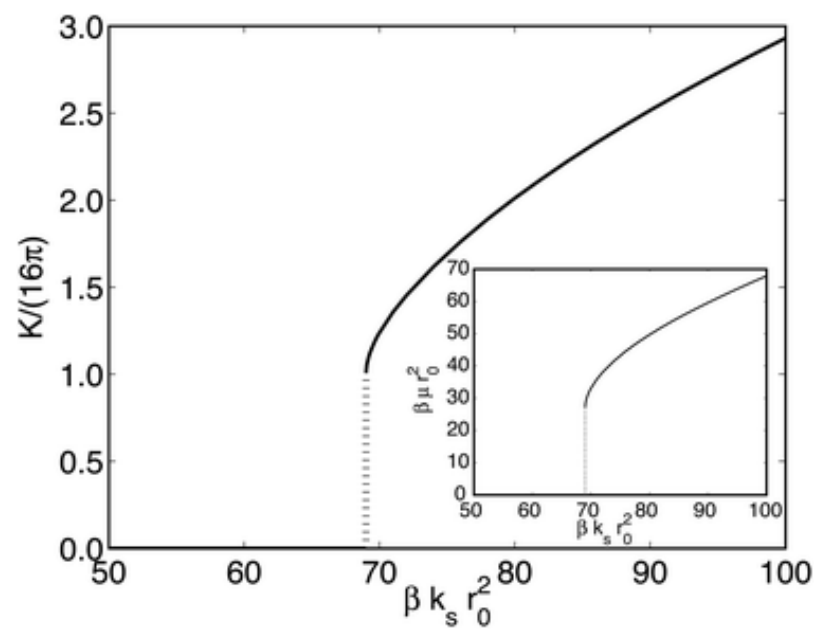

Fig. 7 Renormalized dislocation coupling constant $K_{R}$ versus the stretching modulus $B k_{s} r_{0}^{2}$ (with $k_{b}=$ $\left.k_{s} r_{0}^{2} / 10\right)$, obtained from eqn (6). The coupling constant discontinuously jumps from $K /(16 \pi)=1$ to $K /(16 \pi)=0$ at $B k_{s} r_{0}{ }^{2}=69$, corresponding to a crystalline-hexatic phase transition. Similarly, the shear modulus (inset) decreases with decreasing leg stiffness in the crystalline phase until it vanishes upon transitioning from the crystalline to the hexatic phase.

The phase diagram presented in Fig. 7 provides a prediction for the crystalline-hexatic phase transition for our simulations presented in Fig. 6. Specifically, the transition is predicted to occur between condition C and D in Fig. 6 . To verify the phase behavior in our simulations, we turn to structural correlation functions that have previously been used to determine order-disorder transitions in two-dimensional crystals. ${ }^{32.33}$ In the framework of classical theories of dislocation-mediated melting in two dimensions,,$\frac{32.33}{3}$ appropriate metrics for phase transition are translational- and orientational-order correlation functions.

To facilitate an analysis of the structural order, it is necessary to define an equivalent hexagonal lattice to our honeycomb lattice. Our construction of such a hexagonal lattice involves tagging pinwheels that have leg orientations $\left(0^{\circ}, 120^{\circ}, 240^{\circ}\right)$ in the initial honeycomb state. The equivalent lattice includes only these tagged pinwheels, which make up a hexagonal lattice with spacing $b=\sqrt{3} r_{0}$ that is superimposed over the whole honeycomb structure. Using standard definitions,,$^{48}$ we define primitive translation vectors $\quad \vec{a}_{1}=\sqrt{3} r_{0} \hat{y}, \quad \vec{a}_{2}=\frac{3}{2} r_{0} \hat{x}+\frac{\sqrt{3}}{2} r_{0} \hat{y}$ and reciprocal vectors $\vec{b}_{1}=-\frac{2 \pi}{3 r_{0}} \hat{x}+\frac{2 \pi}{\sqrt{3} r_{0}} \hat{y}, \quad \vec{b}_{2}=\frac{4 \pi}{3 r_{0}} \hat{x}$, resulting in six Bragg peaks $\vec{G}_{\alpha}(\alpha=1,2, \ldots, 6)$ with magnitude $\left|\vec{G}_{\alpha}\right|=\frac{4 \pi}{3 r_{0}}$. With this construction, we convert an initially perfect honeycomb structure to a hexagonal lattice that is more amenable to structural analyses. We use this equivalent hexagonal construction in all of our proceeding calculations.

We express the $i$ th pinwheel position as $\vec{r}_{\mathrm{i}}=\vec{r}^{\left({ }^{(0)}\right.} \mathrm{i}+\vec{u}\left(\vec{r}^{(0)} \mathrm{i}\right)$, where $\vec{r}^{(0)}{ }_{i}$ is the position of the $i$ th pinwheel in the undeformed state, and $\vec{u}\left(\vec{r}^{(0)}\right)$ is the lattice displacement at the position $\vec{r}^{(0)}{ }_{i}$. Note, these definitions are currently applied to the equivalent hexagonal lattice rather than the actual honeycomb structure. The local density is expressed 
using a Fourier representation (i.e. in the reciprocal-vector basis), and the contribution from the six Bragg peaks to the density at the $i$ th lattice point is given by

$$
\rho_{G}\left(\vec{r}_{i}^{(0)}\right)=\frac{1}{6} \sum_{\alpha=1}^{6} \exp \left[i \vec{G}_{\alpha} \cdot \vec{u}\left(\vec{r}_{j}^{(0)}\right)\right],
$$

which is defined to be unity in the undeformed state $\vec{u}\left(\vec{r}^{(0)}\right)=0$. We define the translational-order correlation function as

$$
C_{G}\left(\vec{r}_{i}^{(0)} \vec{r}_{j}^{(0)}\right)=\left\langle\rho_{G}\left(\vec{r}_{i}^{(0)}\right) \rho_{G}^{*}\left(\vec{r}_{j}^{(0)}\right)\right\rangle_{E}
$$

where $\langle\ldots\rangle_{E}$ represents an average over an ensemble of snapshots at the undeformed positions $\vec{r}^{(0)}{ }_{i}$ and $\vec{r}^{(0)}{ }_{j}$. Since this quantity is invariant with respect to a lattice translation and rotation, the translational-order correlation function is expressed as a function of the separation between lattice points, such that

$$
C_{G}(R)=\left\langle\rho_{G}(R) \rho_{G}^{*}(0)\right\rangle
$$

In eqn (9), the undeformed separation $R$ is the distance between two points in the undeformed state, and the average $\langle\ldots\rangle$ now involves an average both over an ensemble of snapshots and over all lattice points whose undeformed separation is equal to $R\left(\right.$ i.e. $\left|\vec{r}^{(0)}-\vec{r}_{i}^{(0)}\right|=R$ ). This representation greatly improves the sampling of this quantity.

The theory of dislocation-mediated melting in two dimensions ${ }^{32-34}$ indicates that the translational-order correlation function in the crystalline phase has the power-law behavior $C_{G}(R) \sim R_{G}^{-\eta}$, where

$$
\eta_{G}=\frac{4 \pi k_{B} T}{9 r_{0}^{2}} \frac{\left(3 \mu_{R}+\lambda_{R}\right)}{\mu_{R}\left(2 \mu_{R}+\lambda_{R}\right)} .
$$

To find $\eta_{G}$, one computes the renormalized Lamé coefficient $\mu_{R}$ and renormalized coupling constant $K_{R}$ using eqn (6) and find $\lambda_{R}$ using the relationship between $K_{R}$ and $\lambda_{R}$ from eqn (5). Fig. 8 illustrates the variation of $\eta_{G}$ with stretching modulus $\beta k_{s} r_{0}^{2}$. As stretching modulus decreases, $\eta_{G}$ increases up to the maximum limit of $1 / 3$ at the crystalline-hexatic phase transition. Further reduction in $\beta k_{s} r_{0}^{2}$ leads to $\eta_{G}$ diverging, corresponding to a change in the behavior of $C_{G}$ from a power-law decay in the crystalline phase to an exponential decay in the hexatic phase. Since the behavior of $C_{G}$ in the crystalline phase decays as a power law with $R$ and the behavior of $C_{G}$ in both the hexatic phase and liquid phase decays exponentially, $C_{G}$ is a useful determinant of the transition from a crystalline phase to the hexatic phase. 


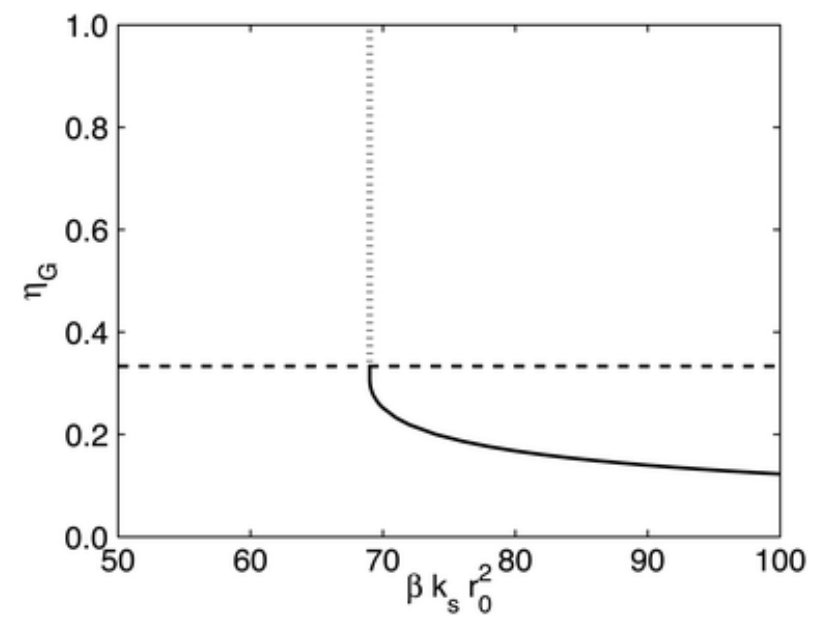

Fig. 8 Exponent $\eta_{G}$ of algebraic decay of translational-order correlation function versus the stretching modulus $B k_{s} r_{0}^{2}$ (with $\left.k_{b}=k_{s} r_{0}^{2} / 10\right)$.

The local orientational order $\psi\left(\vec{r}_{i}\right)$ at the deformed-state position of the $i$ th pinwheel is given by

$$
\psi\left(\vec{r}_{i}\right)=\frac{1}{n_{i}} \sum_{j=1}^{n_{j}} e^{6 i \theta_{\theta}\left(\vec{r}_{i}\right)},
$$

where $\theta_{i j}\left(\vec{r}_{i}\right)$ is the orientation of the line from the equivalent hexagonal lattice site at $\vec{r}_{i}$ to its $j$-th nearest-neighbor site, and $n_{i}$ is the number of nearest neighbors to the $i$ th pinwheel that is defined using a Voronoi diagram ${ }^{49}$ of the pinwheels located at equivalent hexagonal lattice sites. The orientational-order correlation function is defined by

$$
\mathrm{C}_{6}\left(\vec{r}_{i}, \vec{r}_{j}\right)=\left\langle\psi\left(\vec{r}_{i}\right) \psi^{*}\left(\vec{r}_{j}\right)\right\rangle_{E} .
$$

As in $C_{G}$, we define the average over all $i, j$ pairs with a prescribed separation, such that

$$
C_{6}(r)=\left\langle\psi(r) \psi^{*}(0)\right\rangle
$$

where $\langle\ldots\rangle$ involves averaging over an ensemble of snapshots while binning all $i, j$ pairs whose deformed-state separation is $r$ (i.e. $\left.\left|\vec{r}_{i}-\vec{r}_{j}\right|=r\right)$.

Although the translational-order correlation function $C_{G}$ decays for a two-dimensional crystal, the orientationalorder correlation function $C_{6}$ tends to a constant at large separation $r$ for a two-dimensional crystalline phase. ${ }^{32-34}$ The hexatic phase is akin to a two-dimensional liquid crystal, and the local fluid exhibits orientational order. However, the local orientational correlation decays at large distances according to the power-law $C_{6}(r) \sim r_{6}{ }_{6} \cdot \frac{32-34}{\frac{1}{3}}$ The exponent $\eta_{6}$ stays below the maximum limit of $1 / 4$ before transitioning from the hexatic phase to the liquid phase. ${ }^{32-34}$ The transition from a hexatic phase to a liquid phase is marked by the separation of 5-7 defects into mobile 5-member and 7-member disclinations. Within the liquid phase, the orientational-order correlation function decays exponentially with $r$. Therefore, the orientational-order correlation function exhibits three distinct behavior in these three phases: $C_{6}$ in the crystalline phase tends to a constant, $C_{6}$ in the hexatic phase decays as a power law, and $C_{6}$ in the liquid phase decays exponentially. 
Fig. 9 shows the translational-order correlation function $C_{G}$ (Fig. 9A) and the orientational-order correlation function $C_{6}$ ( Fig. 9B) from the 6 simulations presented in Fig. 6, i.e. $\beta k_{s} r_{0}^{2}=85$ (condition A, red), 75 (condition B, cyan), 70 (condition $\mathrm{C}$, magenta), 65 (condition D, green), 60 (condition $\mathrm{E}$, blue), and 55 (condition $\mathrm{F}$, black) and $\beta k_{b}=$ $\beta k_{s} r_{0}^{2} / 10$. The dashed lines in the plot of $C_{G}$ (Fig. 9A) are analytical predictions for the power-law decay $C_{G} \sim R^{-\eta}{ }_{G}\left(\eta_{G}\right.$ predicted from Fig. 8) for the three simulations that are predicted to be in the crystalline phase from the phase diagram in Fig. 7. The crystalline-hexatic transition is predicted to occur at $\beta k_{s} r_{0}^{2}=69$, which is just below the rigidity for condition C. Notably, there is close agreement between the power-law decay from the analytical theory and the simulations for conditions $\mathrm{A}, \mathrm{B}$, and $\mathrm{C}$, and subsequent reduction of the rigidity to condition $\mathrm{D}$ results in a marked change in $C_{G}$ to decaying exponentially. We note a leveling of $C_{G}$ at large separation for condition D (green curve in Fig. 9A), which we attribute to sampling and the finite size of the simulation box. Therefore, the translational-order correlation function $C_{G}$ provides a clear metric for determining the melting point of the lattice, which quantitatively agrees with classical theories of dislocation-mediated melting in two-dimensional crystals. $\underline{31-34}$
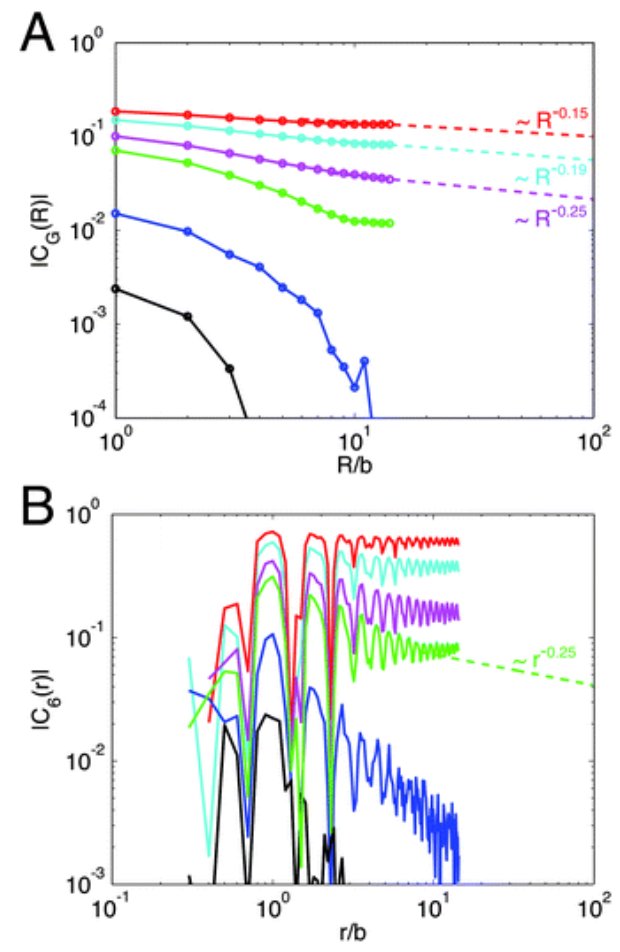

Fig. 9 Panel A gives the translational-order correlation function versus the undeformed separation distance $R / b$ for $B k_{s} r_{0}^{2}=85$ (red), 75 (cyan), 70 (magenta), 65 (green), 60 (blue), and 55 (black) and $B k_{b}=$ $B k_{s} r_{0}^{2} / 10$. Dashed lines (red, cyan, and magenta) are asymptotes $\sim R^{-n}$, indicating $\eta_{G}$ stays below the maximum of $1 / 3$ before transitioning from crystalline to hexatic phase (see text). Panel $B$ shows the variation of the orientational-order correlation function with deformed separation distance $r / b$.

The orientational-order correlation function $C_{6}$ in Fig. 9B includes a plot of the maximum decay behavior in the hexatic phase, $C_{6} \sim r^{-1 / 4}$, which marks the transition from a hexatic phase to a liquid phase. The orientational correlation exhibits a notable transition from condition $\mathrm{D}$ (green) to condition $\mathrm{E}$ (blue). The decay of $C_{6}$ for condition $\mathrm{D}$ exhibits a power-law decay for intermediate $r$ with a decay coefficient $\eta_{6} \leq 1 / 4$; though, $C_{6}$ for condition D in Fig. 9B (green) 
exhibits a similar leveling at large separation as $C_{G}$ for condition D. However, there is a considerable reduction in the orientational correlation between condition D (green) and condition E (blue) in Fig. 9B. For the sampling in our simulations, it is difficult to determine whether conditions $\mathrm{E}$ and $\mathrm{F}$ decay exponentially or as a power law, but the power law decay coefficient would be substantially larger than 1/4 for both condition E and condition F. Therefore, we identify condition $\mathrm{E}$ and condition $\mathrm{F}$ as existing in a disordered liquid phase.

The three phases observed in our two-dimensional model represent very different scenarios of the large-scale physical behavior of a clathrin film, assuming the phase behaviors prevalent in our two-dimensional model persist when the clathrin assembles on a fluctuating membrane. The crystalline phase behaves as a solid elastic material that opposes shear deformation (i.e. $\mu_{R} \neq 0$ ). This property is important in the stabilization of local curvatures; however, this phase does not permit changes in the lattice structure to accommodate different cargo shapes and sizes. The hexatic and liquid phases are fluid in nature, permitting lattice rearrangements via the creation and migration of defects. Although responsive, these phases lack the mechanical integrity to stabilize deformations, particularly to the membrane to which they are attached. The orientational correlation in the hexatic phase implies that the lattice maintains a local crystalline ordering that persists over moderate length scales (multiple clathrin leg lengths). Therefore, the hexatic phase has a locally pre-assembled honeycomb that could seed the formation of cage-like structures. The liquid phase is highly disordered locally, thus lacking pre-seeded structural features. Modulating the elasticity and interactions between clathrin triskelia (and perhaps the local mechanical properties of the membrane) would act to shift the behavior of the lattice, potentially as a mechanism to transition between the aforementioned behavioral scenarios. Our current model is restricted to two dimensions and does not cover out-of-plane processes. However, we anticipate that the fundamental mechanisms for lattice reorganization are similar in two and three dimensions, and our results act as a basis for understanding these large-scale structural dynamics.

\section{Summary}

This work represents the development of a theoretical model to address the thermodynamics and kinetics of clathrin self-assembly. Our model formulation provides a flexible platform to study the roles of molecular elasticity, protein interactions, and thermally induced structural rearrangement in the self-assembly and large-scale behavior of clathrin lattices. We have developed a novel simulation approach that combines Brownian dynamics simulations with dynamic Monte Carlo, facilitating the study of hundreds of clathrin triskelia at sufficient time scales for lattice assembly. We perform energy optimization to study the mechanical properties of clathrin lattices, and Monte Carlo simulations are employed to address the thermodynamic behavior of clathrin systems that are sufficiently large to address structural correlations within the fluctuating lattice. This multi-faceted simulation approach provides a broad range of insights into the physical behavior of our clathrin model.

The dynamics of self-assembly of our clathrin model exhibits an initial clustering followed by a percolated lattice that contains many internal defects that must be alleviated. Propagation of defects to large void spaces results in an ordered final assembly. To further understand these dynamic processes, we address the mechanisms for defect creation and migration. We illustrate that the creation of 5-7 defects in an otherwise perfect honeycomb lattice resembles the 
creation of edge dislocations with equal and opposite Burgers vectors within a crystalline lattice. Leveraging this analogy, we interpret the thermodynamic behavior of our model in terms of established phase behaviors of twodimensional crystalline solids. We show that classical theories of dislocation-mediated melting in two-dimensional crystals can be employed to map out the phase diagram of our clathrin model. This idea is further developed by employing orientational- and translational-order correlation functions as a determinant of the thermodynamic phase of our model.

Our results support a correspondence between our clathrin model and a two-dimensional crystalline solid. Given the crucial role that defects play in two-dimensional melting, the ability of a clathrin lattice to form defects and permit defect migration is a critical determinant of the large-scale response of the lattice. The biological function of clathrin as a responsive material hinges on the ability of the lattice to form closed polyhedral cages that contain a prevalence of 5member ring defects. Our results suggest that modulating the elastic properties of clathrin could lead to a precipitous change in whether the lattice is able to reorganize around an object or maintain a stable structure that opposes elastic deformation.

Our current two-dimensional model is intended to study the fundamental role of elasticity in clathrin lattice reorganization. Further works will extend our theoretical model to address the self-assembly of clathrin into threedimensional nanostructures, either in solution (relevant to in vitro assembly) or on a flexible membrane (relevant to the biological function of clathrin). The mechanisms of defect creation and migration on a membrane are expected to be consistent with the mechanisms discussed in our present manuscript. Therefore, our current work represents a critical step in identifying the dominant physical mechanisms for lattice rearrangement, both in two dimensions as well as in the more biologically relevant case of membrane-bound assembly.

\section{Appendix A: Clathrin Model}

The total energy $E$ in our model is given by

$$
\begin{aligned}
E & =\frac{k_{s}}{2} \sum_{i=1}^{N-1} \sum_{j=i+1}^{N}\left(r_{i j}-r_{0}\right)^{2} L_{i j}-\varepsilon \sum_{i=1}^{N-1} \sum_{j=i+1}^{N} L_{i j} \\
& +\sum_{j=1}^{N} \sum_{\substack{i=1 \\
i \neq j}}^{N-1} \sum_{\substack{k=i+1 \\
k * j}}^{N} \varepsilon_{i j k}^{(b)}\left(\alpha_{j}^{i}, \alpha_{j}^{k}, \alpha_{j}^{\prime}\right) L_{i j} L_{j k} \\
& +k_{r} \sum_{i=1}^{N-1} \sum_{j=i+1}^{N} \frac{H\left(d-r_{i j}\right)}{r_{i j}^{4}}
\end{aligned}
$$

where $r_{i j}=\left|\vec{r}_{i}-\vec{r}_{j}\right|, r_{0}$ is the equilibrium bond length between a bound pair of triskelia, and $N$ is the total number of pinwheels. The stretching modulus $k_{s}$ and bending modulus $k_{b}$ define the elastic properties of the pinwheels, and the repulsive strength $k_{r}$ gives the magnitude of the repulsive interactions. The hardcore cutoff distance $d$ indicates the separation distance where repulsive interactions are turned on, and $H(x)$ is the Heaviside step function. 
The link indicator $L_{i j}$ is equal to 1 if there is a bond between pinwheels $i$ and $j$, and 0 otherwise, and connectivity is reciprocal, such that $L_{i j}=L_{j i}$. Each clathrin pinwheel can only bind up to three other pinwheels; thus, the link indicator must satisfy $0 \leq \sum_{j=1}^{N} L_{i j} \leq 3$ for all $i$. The leg-index indicator $\alpha_{j}^{i}$ identifies the leg number that connects pinwheel $j$ to pinwheel $i, e . g$. if the third leg of pinwheel $j$ connects to pinwheel $i$, then $\alpha_{j}^{i}=3$. The leg-index indicator is set to zero for unbonded pinwheels (i.e. $\alpha_{i}^{j}=0$ if $L_{i j}=0$ ). For any pinwheel $i$, there can be only one pinwheel $j$ that has $\alpha_{i}^{j}=1, \alpha_{i}^{j}=$ 2 , or $\alpha_{i}^{j}=3$. Thus, each pinwheel can bind up to three other pinwheels, and each leg can only connect to one other pinwheel.

The stretching energy [first term in eqn (14)] and binding energy [second term in eqn (14)] only includes contributions from the bonded pairs (i.e. for $i, j$ pairs where $L_{i j}=1$ ). The bending energy [third term in eqn (14)] includes a bending energy $\varepsilon_{i j k}^{(b)}\left(\alpha_{j}^{i}, \alpha_{j}^{k}, \alpha_{j}^{l}\right)$ from the pinwheels $i, j$, and $k$ (with pinwheel $j$ at the vertex), where the leg index $\alpha_{j}^{l}$ is the third leg of pinwheel $j$ that is not part of the $i, j, k$ set (thus, $l \neq i$ and $l \neq k$ ). Only those three-pinwheel combinations that have a bond between pinwheels $i$ and $j\left(L_{i j}=1\right)$ and pinwheels $j$ and $k\left(L_{j k}=1\right)$ contribute to the bending energy.

If the third leg is bound to another pinwheel $\left(\alpha_{j}^{l} \neq 0\right)$, the bending energy is

$$
\varepsilon_{i j k}^{(b)}\left(\alpha_{j}^{i}, \alpha_{j}^{k}, \alpha_{j}^{l} \neq 0\right)=\frac{k_{b}}{2}\left(\theta_{i j k}^{\alpha^{l} \alpha_{j}^{k}}-\frac{2 \pi}{3}\right)^{2},
$$

where ${ }^{\theta_{i j k}^{\alpha_{j}^{\prime} \alpha_{j}^{k}}}$ is the angle between the pinwheels $i, j$, and $k$ (pinwheel $j$ at the vertex). The angle ${ }^{\theta_{i j k}^{\alpha_{j}^{\prime} \alpha_{j}^{k}}}$ is defined in a right-hand sense, e.g. if $\alpha_{j}^{i}=1$ and $\alpha_{j}^{k}=2$, then the angle ${ }^{\theta_{i j k}^{\alpha_{j}^{l} \alpha_{j}^{k}}}$ is defined as the counter-clockwise angle from $\overrightarrow{\mathcal{u}}_{i j}=\left(\vec{r}_{i}\right.$ $\left.-\vec{r}_{j}\right) / r_{i j}$ to $\vec{u}_{k j}=\left(\vec{r}_{k}-\vec{r}_{j}\right) / r_{k j}$.

If the third leg is not bound to another pinwheel $\left(\alpha_{j}^{l}=0\right)$, the bending energy contains bending contributions from the $i, j, k$ set and contributions from the implicit leg, whose orientation minimizes the bending energy. This results in the bending energy

$$
\begin{aligned}
\varepsilon_{i j k}^{(b)}\left(\alpha_{j}^{i}, \alpha_{j}^{k}, \alpha_{j}^{l}=0\right) & =\frac{k_{b}}{2}\left[\left(\theta_{i j k}^{\alpha_{j}^{l} \alpha_{j}^{k}}-\frac{2 \pi}{3}\right)^{2}\right. \\
& \left.+2\left(\pi-\frac{\theta_{i j k}^{\alpha_{j}^{l} \alpha_{j}^{k}}}{2}-\frac{2 \pi}{3}\right)^{2}\right] \\
& =\frac{3 k_{b}}{4}\left(\theta_{i j k}^{\alpha_{j}^{l} \alpha_{j}^{k}}-\frac{2 \pi}{3}\right)^{2},
\end{aligned}
$$

which accounts for all of the leg-bending terms from explicitly-defined bonded legs and implicitly-defined unbonded legs.

\section{Appendix B: dynamic simulation methodology}


The pinwheel equation of motion is given by an overdamped Langevin equation, where the potential and Brownian forces balance the drag force. The drag force used in this work neglects long-range hydrodynamic coupling between pinwheels. Therefore, the equation of motion for the $i$ th pinwheel is given by

$$
\xi \frac{\partial \vec{r}_{i}}{\partial t}=-\frac{\partial E}{\partial \vec{r}_{i}}+\vec{B}_{i},
$$

where $\xi$ is the drag coefficient, $t$ is the time, $E$ is the system energy (defined in Appendix A), and $\vec{B}_{i}$ is the Brownian force vector. According to the fluctuation-dissipation theorem, $\frac{3.36}{6}$ the Brownian force vector is characterized by a Gaussian distribution with mean $\left\langle\vec{B}_{i}(t)\right\rangle=0$ and variance $\left\langle\vec{B}_{i}(t) \vec{B}_{j}\left(t^{\prime}\right)\right\rangle=2 k_{B} T \xi \delta\left(t-t^{\prime}\right) \delta_{i j} \mathbf{I}$.

To capture the binding dynamics, we represent the binding and unbinding processes by a set of reaction equations with configuration-dependent rate constants. For an unbonded $i, j$-pair of pinwheels (i.e. $L_{i j}=0$ ), the binding rate constant $q_{b}\left(i, \alpha_{i}^{i} \mid j, \alpha_{j}^{i}\right)$ governs the instantaneous rate of bond formation between the $\alpha_{i}^{j}$ leg of the $i$ th pinwheel and the $\alpha_{j}^{i}$ leg of the $j$ th pinwheel. To form this bond, both legs that participate in the bond must be free, which requires that $\alpha_{i}^{j} \neq$ $\alpha_{i}^{k}$ for any value of $k \neq j$ and $\alpha_{j}^{i} \neq \alpha_{j}^{k}$ for any value of $k \neq i$. Furthermore, a bond is considered unacceptable if the bond would cross any other bond in the simulation, thus avoiding unphysical frustrated states with crossed bonds. The unbinding rate constant $q_{u}\left(i, \alpha_{i}^{i} j, \alpha_{j}^{i}\right)$ gives the instantaneous rate of bond annihilation, if the $i, j$-pair of pinwheels is currently bonded (i.e. $L_{i j}=1$ ) with the bonded-leg values $\alpha_{i}^{j}$ and $\alpha_{j}^{i}$.

Utilizing the system energy in eqn (14), we compute the binding and unbinding rate constants accounting for energy differences before and after binding and unbinding events. For these rate constants, we choose a Barker form. ${ }^{37}$ Thus, the binding rate constant is given by

$$
q_{b}\left(i, \alpha_{i}^{j} \mid j, \alpha_{j}^{i}\right)=\omega \frac{\exp \left[-\beta \Delta E_{b}\left(i, \alpha_{i}^{j} \mid j, \alpha_{j}^{i}\right)\right]}{1+\exp \left[-\beta \Delta E_{b}\left(i, \alpha_{i}^{j} \mid j, \alpha_{j}^{j}\right)\right]},
$$

if all participating legs are free to bind (available and no crossing arises upon binding); $q_{b}\left(i, \alpha_{i}^{i} \mid j, \alpha_{j}^{i}\right)=0$ otherwise. The unbinding rate constant is given by

$$
q_{u}\left(i, \alpha_{i}^{i} \mid j, \alpha_{j}^{i}\right)=\omega-q_{b}\left(i, \alpha_{i}^{j} \mid j, \alpha_{j}^{i}\right)
$$

if the participating legs are currently bound; $q_{u}\left(i, \alpha_{i}^{i} \mid j, \alpha_{j}^{i}\right)=0$ otherwise. In these definitions, $\omega$ is a fundamental reaction frequency (independent of energy), and $\Delta E_{b}\left(i, \alpha_{i}^{i} j j, \alpha_{j}^{i}\right)=E\left(L_{i j}=L_{j i}=1, \alpha_{i}^{j}, \alpha_{j}^{i}\right)-E\left(L_{i j}=L_{j i}=0, \alpha_{i}^{j}=0, \alpha_{j}^{i}=0\right)$ is the energy difference for making a bond between pinwheel $i$ and $j$ with leg indices $\alpha_{i}^{j}$ and $\alpha_{j}^{i}$.

Since the pinwheel positions and the reaction kinetics are tied together, the pinwheel motion and connectivity are evolved simultaneously by numerical simulation. For the motion of the pinwheels, we integrate eqn (17) utilizing a 4th order Runge-Kutta routine with a discrete time step $\Delta t$, using standard methods for the Brownian forces in the discretetime numerical integration. ${ }^{37}$ The bond connectivity is based on the bonds that are defined at the beginning of each time step. At each time step, we perform dynamic Monte Carlo to determine the bonds that are created and annihilated within that time step $\Delta t$, using rate constants based on the pinwheel positions at the beginning of the time step. Thus, 
the algorithm used in our simulations updates the positions and connections at the end of each time step in order to determine the forces and rate constants for the next time step.

The dynamic Monte Carlo simulation for the time period between $t$ and $t+\Delta t$ is a modification of the Gillespie algorithm for a first-order chemical reaction network. ${ }^{38.39}$ At the beginning of the time step, we find the total rate constant

$$
q_{\mathrm{tot}}=\sum_{i=1}^{N-1} \sum_{j=i+1}^{N}\left[q_{b}\left(i, \alpha_{i}^{j} \mid j, \alpha_{j}^{i}\right)+q_{t i}\left(i, \alpha_{i}^{j} \mid j, \alpha_{j}^{i}\right)\right],
$$

which dictates the overall probability $P(\Delta t)=\exp \left(-q_{\text {tot }} \Delta t\right)$ that a reaction will not take place within the time step $\Delta t$. A random number $\gamma_{1}$ (evenly distributed from 0 to 1 ) is selected, and a reaction is determined to occur if $\gamma_{1}>P(\Delta t)$. The reaction that takes place is selected from the binding probability $p_{b}\left(i, \alpha_{i}^{j} \mid j, \alpha_{j}^{i}\right)=q_{b}\left(i, \alpha_{i}^{i} \mid j, \alpha_{j}^{i}\right) / q_{\text {tot }}$ and unbinding probability $p_{u}\left(i, \alpha_{i}^{i} j j, \alpha_{j}^{i}\right)=q_{u}\left(i, \alpha_{i}^{i} \mid j, \alpha_{j}^{i}\right) / q_{\mathrm{tot}}$. Then, the time of the reaction $t^{\prime}$ is determined to be

$$
t^{\prime}=t-\frac{1}{q_{\mathrm{tot}}} \log \left\{\gamma_{2}\left[\exp \left(-q_{\mathrm{tot}} \Delta t\right)-1\right]+1\right\} .
$$

where $\gamma_{2}$ is a random number, evenly distributed from 0 to 1 .

Additional reactions can still occur between the time period $t^{\prime}$ and $t+\Delta t$. To allow for this possibility, the reaction rate constants are recalculated based on the updated connectivities, and a similar dynamic Monte Carlo step is performed based on these new rate constants for the shortened time step $\delta t=t+\Delta t-t^{\prime}$ (note, $\delta t<\Delta t$ ). This process is repeated for progressively shorter time steps until a dynamic Monte Carlo step is unsuccessful, thus exhausting all possible reactions that stochastically occur within the total time step $\Delta t$.

\section{Appendix C: effective continuum elasticity model}

We define the equilibrium position of the $i$ th pinwheel $\vec{r}^{(0)}$ and the displacement field $\vec{u}(\vec{r})$, resulting in a displaced position of the $i$ th pinwheel $\vec{r}_{i}=\vec{r}^{(0)}+\vec{u}\left(\vec{r}^{(0)}\right)$. The difference vector $\vec{r}_{i j}^{(0)}=\vec{r}^{(0)}{ }_{i}-\vec{r}^{(0)}{ }_{j}$ gives the equilibrium separation between pinwheels in the lattice, which satisfies $\left|\vec{r}_{i j}^{(0)}\right|=r_{0}$ for bonded pairs. We consider a honeycomb structure with stretching and bending energies [given in eqn (14)], and each pinwheel is bonded to three neighbors, resulting in a total binding energy of $-3 \varepsilon N / 2$ (factor of $1 / 2$ avoids double-counting bonds). We neglect the repulsive potential in this derivation, since the honeycomb lattice does not exhibit pinwheel overlap.

To find the effective elastic constants of the lattice, we perturb the stretching and bending energies to lowest order in the displacement field $\vec{u}$. Here, we explicitly perform this procedure for the stretching energy. The stretching energy is conveniently rewritten as

$$
\frac{k_{s}}{4} \sum_{\langle i j\rangle}\left(r_{i j}-r_{0}\right)^{2} \approx \frac{k_{s}}{4 r_{0}^{2}} \sum_{\langle i j\rangle}\left[r_{i j}^{(0)} \cdot\left(\vec{u}\left(\vec{r}_{i}\right)-\vec{u}\left(\vec{r}_{j}\right)\right)\right]^{2},
$$

where the summation over $\langle i j\rangle$ implies a sum over all $i, j$ pairs that are bonded, and we include a factor of $1 / 2$ to avoid double-counting these bonds. The displacement field $\vec{u}\left(\vec{r}_{j}^{(0)}\right)$ is Taylor expanded near $\vec{r}^{(0)}$, and the stretching energy is rewritten as 


$$
\frac{k_{s}}{4 r_{0}^{2}} \int d \vec{r} \sum_{\langle i j\rangle}\left[\vec{r}_{i j}^{(0)} \cdot \frac{\partial \vec{u}(\vec{r})}{\partial \vec{r}} \cdot \vec{r}_{i j}^{(0)}\right]^{2} \delta\left(\vec{r}-\vec{r}_{i}^{(0)}\right) .
$$

Here, we have introduced the delta function to replace $\vec{r}^{(0)}$ in order to perform the summation over the bonded index $j$. The sum over $j$ index has two cases: case 1 with leg orientations $\left(0^{\circ}, 120^{\circ}, 240^{\circ}\right)$ and case 2 with leg orientations $\left(180^{\circ}, 300^{\circ}, 60^{\circ}\right)$. Performing the summation over $j$, accounting for these two cases, gives the stretching energy

$$
\begin{aligned}
& \frac{k_{s}}{3 \sqrt{3}} \int d \vec{r}\left(\frac{9}{8} u_{11}^{2}+\frac{9}{8} u_{22}^{2}+\frac{3}{4} u_{11} u_{22}+\frac{3}{2} u_{12}^{2}\right)= \\
& \left.\frac{k_{s}}{4 \sqrt{3}} \int d \vec{r}\left[\frac{1}{2}\left(u_{11}+u_{22}\right)^{2}+\left(u_{11}^{2}+u_{22}^{2}+2 u_{12}^{2}\right)\right]\right],
\end{aligned}
$$

where $^{u_{i j}=\frac{1}{2}\left(\frac{\hat{a} t_{i}}{\hat{c} r_{j}}+\frac{\hat{a} t_{j}}{\hat{o}_{i}}\right)}$ is the strain tensor, and the discrete lattice is approximated by a continuous medium by

replacing the delta function with the equilibrium density $\delta\left(\vec{r}-\vec{r}_{i}^{(0)}\right) \approx \frac{4}{3 \sqrt{3} r_{0}^{2}}$. In its present form, the stretching energy [eqn (24)] provides values for this contribution to the Lamé coefficients $\lambda$ and $\mu$ for the honeycomb lattice. . $0.41^{4}$ The contributions from the bending energy to the Lamé coefficients are found using an analogous derivation as that of the stretching energy contribution.

\section{Acknowledgements}

We greatly appreciate helpful discussions with Sarah Heilshorn, Nicholas Melosh, Sebastian Doniach, and William Nix. S.M. is supported by Stanford University, School of Engineering. N.C., J.S.Y. and A.J.S. are supported by the Department of Energy, Office of Basic Energy Sciences, Division of Materials Sciences and Engineering, under contract DE-AC02-76SF00515.

\section{References}

1 E. Dujardin and S. Mann, Adv. Mater., 2002, 14, 775-788 Article CAS .

2 A. E. Barron and R. N. Zuckermann, Curr. Opin. Chem. Biol., 1999, 3, 681-687 Article CAS .

3 T. O. Yeates and J. E. Padilla, Curr. Opin. Struct. Biol., 2002, 12, 464-470 Article CAS .

4 S. G. Zhang, D. M. Marini, W. Hwang and S. Santoso, Curr. Opin. Chem. Biol., 2002, 6, 865-871 Article Cas .

5 J. D. Hartgerink, E. Beniash and S. I. Stupp, Science, 2001, 294, 1684-1688 Article CAS .

6 N. Nuraje, I. A. Banerjee, R. I. MacCuspie, L. T. Yu and H. Matsui, J. Am. Chem. Soc., 2004, 126, 8088-8089 Article CAS .

7 S. M. Stagg, P. LaPointe and W. E. Balch, Curr. Opin. Struct. Biol., 2007, 17, 221-228.

8 A. Fotin, Y. F. Cheng, P. Sliz, N. Grigorieff, S. C. Harrison, T. Kirchhausen and T. Walz, Nature, 2004, 432, 573- 
579 Article CAS .

9 M. L. Ferguson, K. Prasad, D. L. Sackett, H. Boukari, E. M. Lafer and R. Nossa, Biochemistry, 2006, 45, 59165922.

10 B. Greene, S. H. Liu, A. Wilde and F. M. Brodsky, Traffic, 2000, 1, 69-75.

11 M. Ehrlich, W. Boll, A. van Oijen, R. Hariharan, K. Chandran, M. L. Nibert and T. Kirchhausen, Cell, 2004, 118, 591-605 Article CAS .

12 W. M. Henne, E. Boucrot, M. Meinecke, E. Evergren, Y. Vallis, R. Mittal and H. T. McMahon, Science, 2010, 328, $1281-1284$.

13 J. Heuser and T. Kirchhausen, J. Ultrastruct. Res., 1985, 92, 1-27.

14 J. E. Heuser, J. H. Keen, L. M. Amende, R. E. Lippoldt and K. Prasad, J. Cell Biol., 1987, 105, 1999-2009.

15 P. K. Sorger, R. A. Crowther, J. T. Finch and B. M. F. Pearse, J. Cell Biol., 1986, 103, $1213-1219$ Article CAS .

16 J. H. Keen, M. C. Willingham and I. H. Pastan, Cell, 1979, 16, 303-312.

17 A. J. Jin and R. Nossal, Biophys. J., 2000, 78, 1183-1194.

18 R. Nossal, Biological and Synthetic Polymer Networks and Gels. Polymer Networks 2004 (Macromolecular Symposia Vol. 227), 2005, 17-25-382.

19 R. Nossal, Biocolloids: American Chemical Society Meeting (Macromolecular Symposia Vol. 219), 2005, 1-8-153.

20 M. L. Ferguson, K. Prasad, H. Boukari, D. L. Sackett, S. Krueger, E. M. Lafer and R. Nossal, Biophys. J., 2008, 95, $1945-1955$.

21 A. Fotin, Y. F. Cheng, N. Grigorieff, T. Walz, S. C. Harrison and T. Kirchhausen, Nature, $2004,432,649-653$.

22 J. D. Wilbur, P. K. Hwang and F. M. Brodsky, Traffic, 2005, 6, 346-350.

23 Rate Processes in Plastic Deformation of Materials, Proceedings of the J.E. Dorn Symposium, ed. J. C. M. Li and A. K. Mukherjee, The Am. Soc. for Metals, 1975.

24 Y. Lansac, M. A. Glaser and N. A. Clark, Phys. Rev. E: Stat., Nonlinear, Soft Matter Phys., $2006,73,041501$.

25 T. L. Chantawansri, A. W. Bosse, A. Hexemer, H. D. Ceniceros, C. J. Garcia-Cervera, E. J. Kramer and G. H. Fredrickson, Phys. Rev. E: Stat., Nonlinear, Soft Matter Phys., 2007, 75, 031802 Article .

26 A. Hexemer, V. Vitelli, E. J. Kramer and G. H. Fredrickson, Phys. Rev. E: Stat., Nonlinear, Soft Matter Phys., 2007, 76, 051604.

27 S. Srivastava, A. Santos, K. Critchley, K. S. Kim, P. Podsiadlo, K. Sun, J. Lee, C. L. Xu, G. D. Lilly, S. C. Glotzer and N. A. Kotov, Science, 2010, 327, 1355-1359 Article CAS .

28 T. D. Nguyen and S. C. Glotzer, Small, 2009, 5, 2092-2098 Article CAS .

29 Z. Y. Tang, Z. L. Zhang, Y. Wang, S. C. Glotzer and N. A. Kotov, Science, 2006, 314, $274-278$ Article CAS . 
30 B. Peng, Z. T. Deng, F. Q. Tang, D. Chen, X. L. Ren and J. Ren, Cryst. Growth Des., 2009, 9, 4745-4751.

31 J. M. Kosterlitz and D. J. Thouless, J. Phys. C: Solid State Phys., 1973, 6, 1181-1203 Article CAS .

32 B. I. Halperin and D. R. Nelson, Phys. Rev. Lett., 1978, 41, 121-124 Article CAS .

33 D. R. Nelson and B. I. Halperin, Phys. Rev. B, 1979, 19, 2457-2484 Article CAS .

34 A. P. Young, Phys. Rev. B, 1979, 19, 1855-1866 Article CAS .

35 H. Nyquist, Phys. Rev., 1928, 32, 110-113 Article CAS .

36 H. B. Callen and T. A. Welton, Phys. Rev., 1951, 83, 34-40 Article .

37 M. P. Allen and D. J. Tildesley, Computer Simulations of Liquids, Clarendon, Oxford, 1989.

38 D. T. Gillespie, J. Phys. Chem., 1977, 81, 2340-2361 Article CAS .

39 D. T. Gillespie, Annu. Rev. Phys. Chem., 2007, 58, 35-55 Article CAS .

40 L. D. Landau and E. M. Lifshitz, Theory of Elasticity, Pergamon, 1986.

41 A. E. H. Love, A Treatise on the Mathematical Theory of Elasticity, Dover, 1986.

42 L. Monette and M. P. Anderson, Modell. Simul. Mater. Sci. Eng., 1994, 2, 53-66.

43 T. Kirchhausen, Curr. Opin. Struct. Biol., 1993, 3, 182-188.

44 R. J. Mashl and R. F. Bruinsma, Biophys. J., 1998, 74, 2862-2875.

45 P. W. Fowler and D. E. Manolopoulos, An Atlas of Fullerenes, Oxford University Press, 1995.

46 D. R. Nelson, Phys. Rev. B, 1982, 26, 269-283.

47 J. Weertman and J. R. Weertman, Elementary Dislocation Theory, Macmillan, 1965.

48 P. M. Chaikin and T. C. Lubensky, Principles of Condensed Matter Physics, Cambridge University Press, Cambridge, 1995.

49 C. B. Barber, D. P. Dobkin and H. Huhdanpaa, Assoc. Comput. Mach., Trans. Math. Software, 1996, 22, 469-483. 